\title{
A POST-GRADUATE CLINICAL LECTURE
} ON

\section{I T R A L S T E N O S I S,} WITH SPECIAL REFERENCE TO RECENT INVESTIGATIONS.

Delivered at the London Hospital

By A. ERNEST SANSOM, M.D., F.R.C.P., ETC., Physician to the London Hospital : President of the Medical Bociety of

In the course of clinical lectures which I have delivered this session I have considered the clinical and pathological signs of mitral stenosis as evidenced by the cases under our observation in this hospital. On this occasion I shall confine myself chiefly to a review of the investigations which have been made in recent years towards the elucidation of some of the problems presented by this form of disease.

We will, in the first instance, examine the patients who will serve as object lessons, and I will in the briefest terms state the circumstances with regard to these which I wish you to note.

CASE 1.-C. M., aged 31, female. From 18 years of age suffered from anæmia, pajpitations, and shortness of breath. Six weeks before admission had hæmoptysis, two attacks. No rheumatic history. Pulserate 73 irregular. Blight thrill at apex. Long purring presystolic bruit, finishing up suddenly at the first sound, which is short and sharp in character ; double shock sound during diastole heard at apex. Pulmonary second sound accentuated. Patient improved very much while in hospital, her chief trouble being indigestion. Temperature normal no albumen in urine : quantity passed normal in amount.

CASE II.-E. P., aged 22, female. Patient'had right hemiplegia. She could not write, but recognised her name. The heart examination showed all the signs of uncomplicated mitral stenosis, presystolic murmur, and thrill, with double second sound. The hemiplegia gradually cleared up, so that on discharge patient could walk well. The patient could cops print, etc , but could not write anpthing original. Intelligible speech was absent: she could only say "No" We had no doubt that this patient when admitted was suffering from embolism of the left middle cerebral artery. Nutrition was re.establisbed in the areas served by all the branches save that supplying the speech centres; in these a destructive lesion persisted.

In the cases shown to you there is no physical clinical sign of mitral regurgitation; the lesion, so far as the evidence indicates, is obstructive only. Tne morbid condition of the mitral orifice is that defined by Duroziez ${ }^{1}$ and other French writers as "pure mitral stenosis." This is said by these observers to be characterised by a slow, gradual, progressive narrowing of the mitral orifice, evidenced:

(I) Anatomically by a funnel-shaped transformation of the mitral valve due to the complete union of the two curtains. the resulting membranous funnel retaining a smooth and polished appearance ; and

(2) Clinically by the following signs: (a) Thrill felt near the apex; (b) a prolonged snoring murmur of low pitch, heard during the ventricular diastole, followed by a short and rougher bruit in the presyetolic period; $(c)$ a reduplication of the second sound.

I am unable to accept these views in their entirety. In the first place, a progressive narrowing of the mitral orifice even in young subjects does not invariably result in the funnel. form of mitral stenosis. For example, Hayden recorded the case of a boy, aged 7 , in whom there was no rheumatic history. but who died from cerebral embolism, the result of mitral stenosis of button-hole form. ${ }^{2}$ In this case Dr. Hayden distinctly stated that the murmur of mitral regurgitation did not exist. It is quite clear that the funnel form of valve change cannot with confidence be predicted in the so-called pure mitral stenosis; conversely the funnel mitral in its typical form I have found in old subjects in the degenerative period of life and associated with fibroid changes.

Now as to the etiology of this slowly developing obstructive disease at the mitral orifice. Duroziez has said that pure mitral stenosis is not a rhenmatic affection. With this statement I can by no means agree. I have never myeelf observed nor found any record of observations indicating that mitral stenosis is a congenital malformation; but in very rare cases I have witnessed examples in which other congenital anomalies of the heart have been in association with $a$ fringe of vegetations around the mitral orifice, and with thickenings which, I could thave no doubt, represented an early stage of mitral stenosis. Congenital malformation is one thing and intrauterine endocarditis is another. The vegetations observed had exactly the character of those of endocarditis of the rheumatic form. Duroziez thought that rheumatic endocarditis always resulted in mitral insufficiency or mitral insufficiency and stenosis combined, and not in pure mitral stenosis. In the one case he said that the mitral orifice was unequal with hard edges often cretaceous, in the form of crescent or slit, whilst in the other there was onlya polishe $d$ funnel ; the first was complicated by disease of the other orifices, the second was uncomplicated, and was as the crater of an extinct volcano. These observations also do not accord with $\mathrm{my}+\mathrm{xperience.} \mathrm{In} \mathrm{my}$ Lettsomian Lectures (1883) I gave reasons for the conclusion that there was an intimate association betw een mitral stenosis and rheumatism, but with its insidious rather than its pronounced forms. Rheumatic fever, especially repeated attacks of the disease, is more frequently attended with mitral regurgitation - the slight, insidious, sometimes clinically indetectable forms of rheumatism by mitral stenosis.

Further experience has but confirmed me in these views. Take for an example the following case :

A girl, R. W., whom we had lnng under observation. At the age of $x$ she came under my care for suffering attributed to her heart. She bad never experienced rheumatic fever, but had growing pains and subfebrile attacks. A presystolic murmur and also a systolic murmur were heard. Bhe was discharged and readmitted a twelvemonth after. Then there were thrill and presystolic bruit with a slight systolic blow. After severe ung complications she died, and the post-mortem examination showed the funvel form of mitral stenosis, with some vegetations having all the characters of rheumatic endocarditis encircling the auriculo ventricular orifice.

The case is only an example of many others in which we have found rheumatic endocarditis in the subjects of funnel mitral, and I think that there is a chain of post-mortem evidence which shows that morbid changes having the exact characteristics of rheumatic endocarditis, bricging about mitral stenosis in greater or less degree, are to be found in subjects who have presented very slight signs of rheumatism or even no signs at all. Clinical evidence goes hand in hand with that of morbid anatomy in demonstrating the rheumatic origin of many cases. I described in my Lettsomian Lectures cases in which, after 1 ypical and undoubted attacks of rheumatism, there were, first, the development of signs of mitral insufficiency, and then, in the course of years, those of the combined lesion or of mitral stenosis pure and simple. In a case very carefully observed by $\mathrm{my}$ then house-physician, Dr. Needham, the physical siggs changed from those of regurgitation to stenosis-plus-regurgitation in fifteen days, and ten days subsequently the systolic murmur of mitral regurgitation had quite disappeared, a typical
presystolic murmur of mitral stenosis and reduplication of the second sound remaining.

In December, 1897, I examined a Jady, aged 26, who presented all the signs of mitral stenosis without regurgitation thrill, prolonged diastolic presystolic murmur, with termination in a sudden tap and reduplication of second sound (so-called) from second left costal cartilage downwards to just within the apex. Three months previously this patient was examined by a competent observer, and then no cardiac lesion was in evidence. Three weeks before my examination a systolic murmur was found at the apex. This patient had never suffered from notable rheumatism, but I knew that the members of her family were rheumatic; one brother had suffered from pericarditis and and another from endo carditis.

From many such considerations I cannot swerve from my opinion that in a very large proportion of cases mitral stenosis, though unaccompanied by any signs of regurgitation, is a rheumatic disease. The endocarditis which produces the lesion is protracted and progressive, giving rise to a comparatively slow formation of fibrous quasi-cicatricial tissue which, under the even pressure of the blood, both on the side of the auricle and on that of the ventricle, tends to form the smooth and even membranous funnel which has erroneonsly suggested a congenital malformation.

Yet I would not assert that the origin of mitral stenosis must be always in rheumatic endocarditis. Dr. Pierre Teissier $^{3}$ has written a valuable memoir containing a condensed report of a large number of cases, in which he maintains that the gradual and progressive narrowing of the mitral orifice is due to tuberculosis. I entirely dis sent from this conclusion, which I consider an extraordinary example of special pleading. Teissier cites my observatione, and says, "Sansom is obliged to recognise the fact that mitral stenosig could develop insidiously without the intervention of rheumatism." I did recognise this fact, and pointed it out most strongly, but Teissier would have been more correct if 
he had quoted me as saying "without the intervention of rheumatism as clinically evidenced." For I showed that the form of endocarditis as observed in the post-mortem examination was usually just that which we find associated with rheumatism. In a very few cases the lesion, or the commencement of the lesion, must have existed in intrauterine life. Obstruction at the mitral orifice was found post mortem in an infant who survived birth only twenty-one hours by Benezerd Smith, by Parrot in one who lived twenty-four hours; and in a case recorded by Gerhardt of an infant only 4 months old. In the majority of cases the lesion is recognised about the age of 14 or 15 years-in women especially at the time of puberty. It is to be remembered that mitral stenosis is much more frequent in the female than in the male. Potain, Landouzy, Broadbent, and others, have pointed out that mitral stenosis in the female is frequently associated with anæmia and chlorosis.

That there is some relation between mitral stenosis and tuberculosis I quite agree. Potain found post-mortem evidence of tuberculosis in 12 cases out of 35 of pure mitral stenosis, a proportion of 35 per cent., and Teissier brings evidence to show that this tigure is under the mark. I have myself found very rapid pulmonary tuberculosis evidenced in a case in which there was a funnel mitral, and I have observed clinically other cases in which tuberculous changes were manifested in the subjects of mitral stenosis. I have not found, however, the coexistence in anything like the proportion quoted by Teissier. Taking the cases in Teissier's abstract with those cited by Percy Kidd and others, I find a total of $3^{1}$ in which the association of mitral stenosis with tuberculous lesions were proved post mortem; of these i i presented also the signs of tricuspid stenor is or of endocarditis affecting the tricuspid valve, and 5 others manifested disease of the aortic valves. Uncomplicated mitral stenosis was present, therefore, in only 16 cases. An interesting case has been recorded by Gerhardt of an infant prematurely born at the eighth month, and dying at the age of 4 months from acute tuberculosis. In this case the mitral alone among the valves of the heart was diseased; it was puckered, and its cordæ tendinæ were fused together and thickened. Teissier agrees with Letulle in the statement that $a$ search in the diseased endocardium of cases of mitral stenosis for the bacillus tuberculosis, or for any specific tuberculous lesion therein has always been fruitless; nevertheless he thinks that an attenuated tuberculosis, acquired or hereditary, is the cause of pure mitral stenosis. My conclusion is very different to this. The gradual development of mitral stenosis from a slow endocarditis, for the most part, but not exclusively. due to rheumatism, causes an insufficient supply to the left ventricle, to the aorta, and to the general arterial system; hence a circulatory starvation which brings about a diminished resistance to the morbid influences of the bacillus tuberculosis. In aortic valvular disease the comparative proneness to tuberculous changes was noted long ago. Traube, observing the frequent association between aortic insufficiency and the development of tubercle, limited the law of antagonism between valvular diseases of the heart and tubercle which had been formulated by Rokitansky to mitral disease. An a fortiori argument obtains, therefore, when there is the combined lesion of mitral and aortic disease. A still more marked predisposition exists when there is the combination of mitral and tricuspid stenosis. In tricuspid stenosis there is, of course, an impeded blood supply to the pulmonary artery. Our experience teaches that the subjects of stenosis of the pulmonary artery almost universally die of tuberculosis, and tricuspid stenosis means impeded pulmonary blood supply or obstruction of the pulmonary artery thrown back only one stage.

The practical clinical significance of these observations appears to me to be the following: In any case presenting the physical signs of mitral stenosis the probability of the existence of or the advent of tuberculosis must be duly weighed. Some such cases present signs of marked anæmia, others incomplete development or faulty nutrition. In these the probability of tubercle is greater. Many cases of mitral stenosis, however, show signs which may be easily mistaken for those of pulmonary tuberculosis. They may manifest infraclavicular dulness with râles and auscultatory signs of lang consclidation; by no means infrequently there is hæmoptysis, and hectic fever may accompany these conditions. Yet the signs and symptoms may be entirely independent of tubercle and due to embolisms of branches of the pulmonary artery, to which the subjects of mitral stenosis are prone. $A$ case which simnlates phthisis pulmonalis when mitral stenosis concurs is not of the grave significance of a case in which mitral stenosis is absent. The favourable prognosis must not be hazarded until a complete examination of the sputum has been made and bacilli have or have not been found.

We will now turn to a type of mitral stenosis in which there is no evidence of the intervention of rheumatism in any form-this is the form termed by Huchard, "the mitral obstruction of arterio-sclerosis." Goodhart found on an analysis of the post-mort $\mathrm{m}$ records of 192 cases in which there were post-mortem evidences of granular kidney that about one-fourth of the whole number presented thickenings of the mitral valves or stenosis of the mitral orifice. G. N. Pitt found from an examination of the records of the post-mortem department of Guy's Hospital that the cases of mitral stenosis in the subjects of granular kidneys were to those who did not manifest this form of renal lesion in the proportion of three to one. I have described 9 case in a woman, aged 52 , in whom there was a gradual fibroid transformation in many situations, including a sclerosis of the mitral valve with obstruction of the orifice. Huchard's pupil, Frederic Auguste Blind, ${ }^{4}$ has well described a typical case, and has summarised the evidence, which appears convincing, that this form has a definite nosological position. In this variety the disease commences gradually in persons, more frequently males than females, at or after middle age. There are often signs of degeneration of the arterial walls and the left ventricle is hypertrophied or dilated and hypertrophied; in some cases the physical signs as regards the heart are typical of mitral stenosis, but in many a sudden and loud first sound or a slight systolic localised blowing murmur are all the auscultatory signs observed.

From the evidence which I have examined, therefore, I believe that the most frequent cause of mitral stenosis is a slowly developing endocarditis of the rheumatic form and another cause far less frequent, but one operating for the most part in advanced life, namely, a general or widely diffused, slowly developing fibrosis. I recognise, however, that there may be other forms of obscare etiology, though I find that the hypothesis of any tuberculous causation is not supported by anything like sufficient evidence.

It has been shown that in mitral stenosis of the funnel form there has been in some cases a s7stolic murmur heard in the neighbourhood of the apex. Exceptionally the presystolic murmur is not heard ; only a short systolic murmur is audible. It is the opinion of Goodhart and of Potain that this systolic murmur may be due not to mitral but to tricuspid regurgitatation Dr. D. W. Samways ${ }^{6}$ has made an inquiry concerning this point by examining the post-mortem record of 70 cases examined at Guy's Hospital. In 40 of these a systolic murmur had been audible during life, and in 15 the situation of audibility of the murmur suggested a tricuspid causation. Dr. Samways considers that it is reasonable to conclude that in cases of mitrul stenosis, regurgitation through the mitral aperture is less common than has been supposed, a tricuspid reflux having often been mistaken for a mitral regurgitation.

We come now to a very interesting question. Finding, as we do, that in the so-called pure mitral stenosis there is no clinical evidence of mitral regurgitation, how is such regurgitation prevented? In the search for an answer to this question, our attention should first be called to the action of the left auricle in mitral stenosis. The lessons of morbid anatomy are to the effect that in some cases of this affection the muscular tissue of the auricle is enormously hypertrophied, in others atrophied to almost complete extinction. I have myself observed post mortem in a child the auricular muscle as thick as that of the right ventricle, and in an aged woman an extreme dilatation of the auricle, the cavity being lined with laminated clot, and in the thin wall muscular fibres scarcely represented at all.

An excellent summary concerning the left auricle in this disease has been published in the graduation thesis of $M$. Gérard. ${ }^{6}$ Some authors -as Laennec, Stokes, and Niemeyer 
-have concluded that dilatation of the left anricle is the direct consequence of mitral obstruction. Others-as Bouilland, Potain, and Rendu-have insisted that dilatation and hypertrophy go together: "the left auricle dilates and hypertrophies simultaneously" (Potain and Rendu). The records of post-mortem examinations, however, have not at this stage of the inquiry determined the exact relation of the hypertrophy and dilatation.

First, Is dilatation of the auricle a necessity in compensated mitral stenosis? Is hypertrophy always to be demonstrated ? Is hypertrophy of the auricle a provision for effecting and maintaining compensation? Are dilatation of the auricle and atrophy of the muscle late effects- the latest sign of a falling compensation? Gerrard says: "In pure mitral stenosis, the heart adapting itself, as it were, naturally to a diminished labour, lesions of the left auricle are often absent for a long period." This is contrary to what occurs in the combined lesion of insufficiency and stenosis, where the adaptation of the heart is impossible and hypertrophy of the left auricle occurs at any rate provisionally.

The evidence of morbid anatomy has been further investigated by Dr. Samways. ${ }^{8}$ Examining the records of 70 postm ortem examinations of cases of mitral stenosis made at Guy's Hospital, he finds that in pronounced mitral stenosis the characteristic modification of the left auricle is not dilatation but hypertrophy, and though at the post-mortem examination dilatation is often associated with hypertrophy the dilatation is probably not evident during life whilst compensation exists, and it occurs when compensation fails.9

Let us turn to clinical evidence. I examined a large number of cases of mitral stenosis by means of the cardiograph. In 1880 I published some cardiographs showing a very pronounced, in some cases enormous, elevation which could only indicate an inordinately powerful contraction of the auricles in these cases. ${ }^{9}$ In my work on the Diagnosis of Diseases of the Heart, published in 1892 , I adduced arguments which seemed to me to point convincingly to the conclusion that the muscular contraction of the left auricle in some cases of mitral stenosis was sufficiently powerfal to lift the apex of the left ventricle. Dr. Dickinson, who opposed the views of Fauvel, Gairdner, and a large number of other clinical observers as to the causation of the so-called presystolic murmur, adduced careful and accurate observations to show that in some cases the murmur which was observed in confirmed mitral stenosis occurred not before, but simultaneously with, the elevation of the heart's apex, as perceived by the finger. Dr. Dickinson's inference was that the murmur which was heard could not be presystolic and of auricular causation, but systolic and the work of the left ventricle. I contended that Dr. Dickinson's inference was unsound, though his clinical observations were strictly accurate. I arged ${ }^{10}$ that one should not assume that the elevation of the heart's apex as felt by the finger of the observer is of necessity synchronous with the contraction of the ventricle-that in some cases the hypertrophied auricle extruding its blood contents through a moderately narrowed mitral orifice might be sufficiently powerful itself to cause a pronounced elevation of the apex, the proper systole of the ventricle following.

My observations and inferences thus expressed have been exactly corroborated by Professor Potain of Paris, who, in his work in the Clinique Médicale de la Charité, 1894, has recorded a number of cardiographic and sphygmographic Investigations of his own, and has collated the results of others who have employed instruments of precision. Potain agrees with Dickinson that the presystolic roll (roulement presystolique) coincides with the apical impulse. But this lifting of the apex is not always produced by the ventricular systole, but often wholly or in great part by the systole of the auricle and the distension of the ventricle which this determines. The elaborate reasonings on which this conclusion is based are detailed in the article on the apex beat of the heart. ${ }^{11}$ Potain further states that accumulated proofs leave no room for doubt not only that the left auricle contributes to the impulse of the left ventricle, but that the contraction of the muscle of the auricle does not cease until the complete closure of the mitral orifice, and the commencement of the propulsion of the blood of the left ventricle into the aorta. Dr. Samways has seen in this an explanation of the preven- tion of regurgitation into the left ventricle in the "pure". form of mitral stenosis. He contends, from experimental as well as mathematical data, that the auricular cavity being small (its muscular walls by their contraction having nearly emptied it) and the ventricular cavity being large (nearly replete), it is possible that the auricle as a contracting sphere may be master of the situation, and force the remnant of its contents into the ventricle, in spite of the muscle of the latter being already in contraction.

Dr. Robert H. Woods of Dublin had already shown in 1892 by mathematical demonstration that a small contracting sac has a mechanical advantage over a large one. Dr. Woods, ${ }^{12}$ however, has expressed his opinion that Dr. Samways pushes the matter to an absurdity when he contends that the left auricle by its contraction sends blood into the contracting ventricle. This question is not to be judged only from the standpoints of experimental physics and mathematical formulæ. I have considered it from the side of pathology and morbid anatomy and $I$ think that the contentions of Professor Potain and Dr. Samways are fully justified.

I cannot regard the auricle only as a contracting sphere. It is not a muscular sac of uniform contractile power throughout. The commencing phase of the emptying its cavity is in the pulmonary veins, the concluding in its most muscular part, the auricular appendix. Moreover, there is another cause at work to effect the closure of the mitral orifice-the diminution of the area of the auriculo-ventricular ring and the shortening and approxination of the papillary muscles. Marc Sé $e^{13}$ has contended that the three papillary muscles of the left ventricle in their contraction are so disposed as to fit into each other and so contribute to close the upper part of the ventricle. It is true that these muscles do not contract at the very earliest period of systole of the ventricle; the parietal muscle commences, but is reinforced by the papillaries in an extremely brief time. ${ }^{14}$ I only adduce these considerations to show that the contraction of the auricle is not to be considered as the sole force, but is an auxiliary force in preventing regurgitation at an early part of ventricular systole. Furthermore, if the auricle can contend successfully with the ventricle until such moment as the latter has overcome the pressure in the aorta sufficiently to open the aortic valves, this is all that the argument demands. Auricle and ventricle would co-operate to do this. Dr. Robert $H$. Woods makes a statement with which I by no means agree. I know the view is held by many, and I think it time that the evidence of the other side should be duly considered. He writes: "There are novalves between the auricle and the lungs, and therefore raising the intra-auricular pressure during contraction of the ventricle could only result in the blood following the path of least resistance, namely, back into the pulmonary veins." But there is a valvular mechanism between left auricle and lungs-namely, in the pulmonary veins themselves. "At the moment of the auricular systole the orifices of the pulmonary veins are narrowed in the contraction of the layer of muscular fibres by which they are surrounded, and any great reflux of the blood into the veins is thus prevented." 15 "The contraction of the circular muscular fibres around the venous orifices and the subsequent contraction of the auricle cause these ve!ns to empty themselves into the auricle; and by their presence and action they prevent any large quantity of blood from passing backwards into the veins when the auricle contracts. No valves are present in the superior and inferior cavæ in the adult heart, or in the pulmonary veins; hence the contraction of these circular muscular fibres plays an important part in preventing any reflux of blood during the contraction of the auricles." 18

From these observations you will infer that though there is no membranons valve there is a very efficient sphincter-and a sphincter may be more powerful than a valve tending to prevent reflux towards the lungs. I think that this very important quasi-valvular arrangement is too often overlooked. It may be said that the sphincters may become inefficient. No doubt they may; hence some of the lung complications of the late stages of mitral stenosis. As evidence of the strain to which the pulmonary veins are subjected, I may cite the observations of Dr. James Barr, of Liverpool, who found well marked atheroma of the pulmonary veins in cases of mitral stenosis.

There are circumstances observed in cases of mitral stenosis 
in children and young persons which have seemed to me to point very strongly to the correctness of the inferences of Potain and Samways. It is the rule to find in the post-mortem examination of such cases that the left ventricle is of small capacity contrasting with the large dilated right ventricle of the same subject, and with the normal left ventricle of a child of the same age as the subject observed. There cannot be any doubt that the morbid condition of mitral stenosis is of slow pathogenesis. These morbid processes must have commenced at the periphery of the valve curtains, or at the auriculo-ventricular orifice, and it seems to me that unless some cause prevented regurgitation, regurgitation must have occurred. If such regurgitation had occurred, why is it that its usual consequences are not in evidence? Surely the enhanced blood pressure within the ventricle would have caused left ventricular dilatation. But such dilatation is conspicuous by its absence. It is in the hlghest degree probable therefore that regurgitation was pre. vented, and, according to the accumulated testimony, prevented by the action of the auricle.

It is probable that the normal endowment of the auricular muscle is sufficient to prevent the regurgitation in the earlier stages of development of mitral stenosis, but in course of time the muscle becomes hypertrophied. Later on the dilatation of the auricle preponderates, and the muscular fibres become attenuated to almost extinction. Atrophy of the anricular muscle may, however, occur in the earlier stages from various causes of faulty nutrition. My own experience coincides with that of Dr. Samways in establishing as a general rule the doctrine that " the characteristic alteration in the left auricle in mitral stenosis is not dilatation but hypertrophy-dilatation is probably introduced only with the auricular breakdown."

The prognosis in the condition of disease which we are considering is grave. I have found the average age at death of 6r patients to be 32.7 years, but it is to be remembered that my cases included an unusual number of children, for in the etiology of the disease in children I was much interested, and probably more than my fair proportion of cases were sent nnder my notice. The late Dr. Hayden's cases, 42 in number, gave 37.8 years. Sir William Broadbent, 53 cases, gives as the average age at death 33 years for males and 37 to 38 for females.

The most recent inquiry, that by Dr. Samway $8^{17}$ as to the post-mortem records of Guy's Hospital during ten years shows that the average age at death, males and females (identically) is $38 \frac{1}{3}$ years; the less pronounced forms of constriction of the orifice 43.6 years ; the more extreme stenosis 336 years.

The concurrence of testimony, therefore, is to show that the conditions of the disease are so grave that life is rarely prolonged beyond 40 years. In the form of disease which is associated with chronic fibrosis and granular kidney, however, the age at death is much more advanced; but these commence insidiously for the most part at a comparatively advanced age. I have observed such a case at the age of 70 .

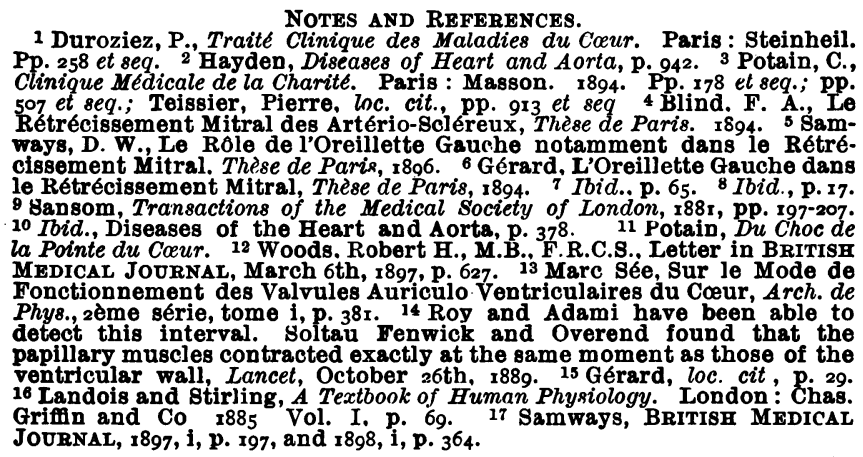

Thr lepers of the Orange Free State are in part placed on $a$ farm in the neighbourhood of Bloemfontein, in part maintained on Robben Island at the expense of the State. A proposal is now made that the lepers should be removed from the island and placed upon another farm in the Free State.

\section{THE CROONIAN LECTURES}

ON THE

CHEMICAL PRODUCTS OF PATHOGENIC BACTERIA CONSIDERED WITH SPECIAL REFERENCE.

TO ENTERIC FEVER.

Delivered before the Ryyal College of Physicians of London;

BY SIDNEY MARTIN, M.D., F.R.S., F.R.U.P.,

Professor of Pathology, University College; Assistant.Physician to. University College Hospital and to the Hospital for Consumption,
Brompton.

LECTURE II.

WIтH these preliminary observations we now pass to the consideration of the investigation more recently made on the chemical products of the typhoid bacillus, of the bacillus coli communis, and of a form allied to these, namely, the bacillus enteritidis of Gärtner.

These three micro-organisms are in culture closely allied to each other. They all grow in broth, forming a cloudiness: and a sediment, not commonly a scum on the surface, although this is present sometimes in cultures of the bacillus coli. The growth on agar is very similar in all three forms, being more fluorescent with the bacillus coli than with the other two forms. On gelatine they grow without producing any liquefaction of the medium. Their differences are as follows: In glucose gelatine and glucose agar the bacillus coli and Gärtner's bacillus form numerous bubbles of gas, while the typhoid bacillus, although it grows copiously, produces no bubbles. The bubbles are due to the formation of gas from the sugar present. On potato the bacillus coli gives a fleshy yellowish-brown growth; the Gärtner bacillus a slightly coloured, and the typhoid bacillus gives a filmyalmost transparent-growth. Milk is coagulated by the bacillus coli, but is not coagulated by the typhoid bacillus or by Gärtner's bacillus. Indol is formed in a broth culture by the bacillus coli, not at all by the typhoid bacillus, and only a slight indol reaction can be obtained, and that not constantly, in cultures of the Gärtner bacillus. Some varietles of the bacillus coli do not appear to form indol.

These cultural characteristics are slight, but are sufficient. for the purposes of diagnosis, inasmuch as the bacillus coli can always be distinguished from the other two by the gas it forms in glucose gelatine, by its coagulation of milk and by the indol it produces, whereas Gärtner's bacillus is distinguished from the typhoid bacillus by the fact that it forms gas in glucose gelatine. In any of the media the growth of the bacillus coli and of Gärtner's bacillus is much more copious than that of the typhoid bacillus. The bacillus coli is further distinguished from the typhoid bacillus by not giving: the " serum reaction" with the blood of a typhoid patient or with typhoid "antitoxic serum." Dr. Durham states that Gärtner's bacillus sometimes gives a sedimenting reaction with typhoid blood or serum. All these organisms are flagellated, the typhoid bacillus containing the most numerous flagella, the bacillus coli the smallest number, and Gärtner's bacillns being intermediate. The close similarity : in vegetative properties of these three micro-organisms rendered it advisable that they should be investigated together. They probably play very different rôles in the production of disease. The typhoid bacillus is the cause of enteric fever; the bacillus coli plays a great part in the production of peritoneal lesions proceeding from the intestine and inflammatory conditions connected with the pelvic and other abdominal organs; and the Gärtner's bacillus was originally found in the body of a man who had been poisoned by beef which also contained the bacillus. Dr. Durham. thinks that it may be the cause of some cases of food poison-. ing. Both the typhoid bacillus and Gärtner's baeillus may therefore be considered as micro-organisms, chiefly infecting the intestinal tract, and the bacillus coli-though it is not known to produce any gross lesion in the intestinal tract-is: a natural habitant in that region and may aid in intestinar infections.

The Typhoid Bacillos.

The typhoid bacillus is found in enteric fever, in the intes... 